\title{
Preface: Postmigrantische Stadt? Urbane Migrationsgesellschaften als Ausgangspunkt für einen kritisch-normativen Perspektivwechsel in der sozialgeographischen Stadtforschung
}

\author{
Karin Wiest \\ Leibniz-Institut für Länderkunde, Schongauerstr. 9, 04328 Leipzig, Deutschland \\ Correspondence: Karin Wiest (k_wiest@ifl-leipzig.de)
}

Published: 5 November 2019

\section{Konflikthafte Neujustierungen von Zugehörigkeit und Differenz in der Migrationsgesellschaft}

Weltweite ökonomische Verflechtungen, multilokale Alltagspraktiken, kulturelle und nationale Mehrfachzugehörigkeiten sind grundlegende Kennzeichen mobiler Gesellschaften unter Globalisierungsbedingungen. Sie prägen das Zusammenleben im Alltag auf vielfältige Art und Weise, sei es in konkreten Arbeitswelten, in Bildungsinstitutionen oder Freizeitzusammenhängen. In urbanen Zusammenhängen ist dies meist offensichtlicher als in ländlichen Räumen - so erscheint Migration oft als Thema, das vor allem auf städtischem Terrain ausgetragen wird (u. a. Hess und Lebuhn, 2014b; Y1ldı, 2011). Gleichzeitig ist der Umgang mit internationaler Migration und Diversität durch widersprüchliche Alltagspraktiken und Politiken gekennzeichnet. So werden einerseits neue, umfassende gesellschaftliche Einschlüsse und Zugänge auf unterschiedlichen administrativen Ebenen gefordert, die eine zentrale Grundlage gleichberechtigter gesellschaftlicher Teilhabe bilden. Andererseits werden neue Formen der Ausgrenzung praktiziert, die Marginalisierungen und gesellschaftliche Diskriminierungen (re-)produzieren. Die Zuspitzung der Debatten im Kontext migrationsgesellschaftlicher Realitäten hat sich in jüngster Zeit besonders im Erstarken rechtspopulistischer Bewegungen sowie in identitätspolitisch aufgeladenen Diskussionen wie insbesondere im Umgang mit Fluchtmigration gezeigt. Den Forderungen nach einer größeren Kontrolle und Regulation von Zuwanderung stehen zunehmend emanzipatorische Gegenbewegungen gegenüber, mit dem Ziel, ethnisierende oder kulturalisierende Zuschreibungen zu überwinden und Grenzen sowohl im nationalstaatlichen als auch im sozialen Sinn aufzuge- ben (u. a. Ataç et al., 2015; Hill und Yıldız, 2018; Römhild, 2017). Konflikthafte Auseinandersetzungen um natio-ethnokulturelle Zugehörigkeiten sind nicht zuletzt im Kontext weltweit zunehmender sozioökonomischer Disparitäten zu betrachten. In ihrer produktiven Form können entsprechende Konflikte Integrationsprozesse voranbringen und neue Chancen für das soziale Zusammenleben eröffnen (Spielhaus, 2014; El-Mafaalani, 2018). Grundsätzlich rücken sie die Frage in den Mittelpunkt, welche gesellschaftlichen Strukturen und Mechanismen sozialer Ungleichheit unter dem Stichwort „Migration“ verhandelt werden - ,denn die soziale und kulturelle Reproduktion von Ungleichheiten wird durch Ethnisierungen und Rassifizierungen, durch Religion, Geschlechterdifferenzierungen und durch nationale Identifikation legitimiert" (Çağlar und Glick-Schiller, 2011:150). Oder wie es Naika Foroutan formuliert: „Migration ist nur die Chiffre, hinter der sich vielfältige Konflikte im Umgang mit Pluralität verstecken.“ (Foroutan, 2018:21) Das Hinterfragen und die Dekonstruktion von gesellschaftlichen Zuschreibungen, die oft pauschalisierend mit der Kategorie „Migration“ verknüpft sind, repräsentieren somit ein wesentliches Moment einer sozialwissenschaftlichen Forschung, die der wachsenden Bedeutung transnatio-ethno-kultureller Lebenswirklichkeiten Rechnung trägt. Im Kern geht es hier um die vielfältigen gesellschaftlichen Konstruktionen eines „Anderen“, die in politischen Diskursen und gouvernementalen Praktiken ebenso wirksam werden wie im Alltag (Amelina, 2017; Mecheril, 2018). Eine wesentliche Herausforderung ist es hier zum einen, die komplexen sozialen Konstellationen und Strukturen transnationaler globaler Gesellschaften adäquat zu erfassen, sowie die damit verbundenen Verschiebungen in den Zuschreibungen von gesellschaftlicher Zugehörigkeit 
und gesellschaftlicher Ausgrenzung in den Blick zu nehmen. Zum anderen kommt lokalspezifischen und zeitlichen Ausprägungen im Umgang mit Migration und Diversität eine entscheidende Rolle zu. Pott weist zu Recht darauf hin, dass in der Migrationsforschung bislang kaum thematisiert wurde, dass sie ,ihren Gegenstand erst durch die Nutzung der Raumkategorie, durch die von ihr betrachteten und konstruierten Räume gewinnt“" (Pott, 2018:112).

Vor dem Hintergrund der skizzierten Debatten richtet sich das vorliegende Themenheft auf die Frage, wie „Migration“ als Chiffre für gesellschaftliche Zugehörigkeit und Differenz und „Stadt“ als Ort alltäglicher Routinen und Praktiken der Raumaneignung und Raumproduktion auf unterschiedlichen Ebenen miteinander in Beziehung treten. Dabei soll ein heuristischer Zugang zur Diskussion gestellt werden, der sich im Schnittfeld von Wissenschaft und Praxis, Analyse und politischer Intervention verortet und für sich den Anspruch erhebt, auf Ursachen und Wirkungen gesellschaftlicher Missstände aufmerksam zu machen. Dieser als ,postmigrantisch“ bezeichnete Zugriff wird im Rahmen des vorliegenden Special Issues im folgenden Sinn interpretiert: Er zielt zum einen darauf ab, mehrheitsgesellschaftliche Vorannahmen in den gängigen Untersuchungsfragen der sozialwissenschaftlichen Stadtforschung aus einer dezidiert migrationsgesellschaftlichen Perspektive heraus kritisch zu hinterfragen. Zum anderen stellt er die Wirkungen hegemonialer gesellschaftlicher Konstrukte und Machtverhältnisse auf urbane Gesellschaften zur Diskussion - und zwar auf jene (urbanen) Gesellschaften, die sich im Zuge internationaler Mobilität und Vernetzung beständig verändert haben und neue Realitäten des urbanen Zusammenlebens hervorgebracht haben.

Im ersten Abschnitt dieses Editorials wird zunächst auf die allgemeinen konzeptionellen Herausforderungen an eine Gesellschaftsanalyse unter hochmobilen und global vernetzten Bedingungen eingegangen. Auf dieser Grundlage und im Hinblick auf die mittlerweile zahlreichen Ansätze in diesem Arbeitsfeld werden die wesentlichen Merkmale und ein potenzieller Mehrwert der postmigrantischen Perspektive skizziert.

Im zweiten Abschnitt werden raumbezogene Implikationen eines postmigrantischen Zugangs in den Blick genommen, die darauf abzielen, der Bedeutung von Migration nicht nur als gesellschaftsgenerierender Kraft, sondern auch als einem Faktor des „Place-Makings“ gerecht zu werden. Bezogen auf Untersuchungsgegenstände der Stadtforschung sind diesbezüglich machtkritische Ansätze, die die Bedeutung nationaler Bezüge zugunsten der Betonung globaler Zusammenhänge hinterfragen, besonders grundlegend. Ansätze im Feld der postkolonialen Stadtforschung und der Studien zu „Urban Citizenship“ nehmen hier einen prominenten Platz ein.

Im letzten Abschnitt wird ein Ausblick auf die Beiträge des Themenheftes gegeben, die sich der Aufgabe stellen, die Relevanz einer postmigrantischen Perspektive für Fragestellungen der geographischen Stadtforschung und auf der
Grundlage empirischer Fallstudien auszuloten. Sie zeigen sowohl den heuristischen Mehrwert als auch spezifische konzeptionelle Anforderungen und Umsetzungsschwierigkeiten auf, wenn das „Postmigrantische“ als Beobachtungsfokus für das urbane Zusammenleben gewählt wird.

\section{Migrationsforschung als Gesellschaftsanalyse}

Im Zuge der skizzierten gesellschaftlichen Dynamiken werden gegenwärtig unterschiedliche Forschungsperspektiven und Konzepte diskutiert, die den Wandel von Einwanderungsgesellschaften und die lebensweltlichen Dimensionen gesellschaftlicher Diversifizierung in das Zentrum ihrer Analysen stellen. Als prominente Beispiele seien Arbeiten der Transnationalismusforschung (u. a. Glick-Schiller et al., 1995), Konzepte der Konvivialität (u. a. Wise und Noble, 2016), der Superdiversität (u. a. Vertovec, 2007, 2017; Meissner, 2015) und der Intersektionalität genannt. Der Aufschwung dieser Ansätze, deren zentrales Anliegen es ist, essentialistische, ethnisch-fixierte Forschungsperspektiven und einen methodologischen Nationalismus zu überwinden (u. a. Glick-Schiller et al., 2006), ist - bei aller Unterschiedlichkeit - vor allem eine Folge des Erklärungsverlustes von Konzepten, die sich auf lineare Vorstellungen von Integrationsprozessen beziehen oder versuchen, gesellschaftliche Erklärungen auf die kulturelle oder nationale Herkunft von Migranten zurückzuführen. Ziel entsprechender Ansätze ist es daher weniger, auf eine größere Differenziertheit der sozialen Unterschiedlichkeiten und Herkünfte von Stadtbewohnerinnen und Stadtbewohnern abzuheben, sondern vielmehr die Beziehungen und Wechselwirkungen von sich überlagernden sozialen, kulturellen und ökonomischen Faktoren unter globalen Bedingungen in das Zentrum der Betrachtung zu stellen (Vertovec, 2007:1025; Meissner, 2015). Neben der gemeinsamen Stoßrichtung, nämlich essentialistische Reduktionen und Stereotypisierungen zu überwinden, ist auch die konzeptionelle Neuausrichtung hin zur Untersuchung von sozialen Beziehungen, Machtkonstellationen und gesellschaftlichen Positionierungen grundlegend (Berg und Sigona, 2013; Labor Migration, 2014; Vertovec, 2017). Eine Forderung, die sich vor allem aus der Kritik an einem nationalstaatlich begründeten Migrationsdifferenzparadigma ableitet, ist, dass die Migrationsforschung von ihrem letztendlich immer an eine nationalstaatliche Logik gebundenen Fokus auf Migration abrückt. In entsprechend konsequenter Umsetzung wäre sie dann in eine übergreifende Sozialtheorie einzugliedern, ,,allerdings eine, die gleichzeitig Migration und Ethnizität als wichtige Faktoren in ihre Analyse mit einbezieht“ (Dahinden, 2016:22). Eine solche als ,postmigrantisch“ zu verstehende Sichtweise begreift Gesellschaft konsequent als von Migration beeinflusst und durchdrungen (u. a. Dahinden, 2016; Römhild, 2015). Diese Perspektive soll im Folgenden hinsichtlich ihres normativ-kritischen An- 
liegens und der sich daraus abzuleitenden analytischen Konsequenzen genauer betrachtet werden.

\subsection{Das „Postmigrantische“ - Appell für einen gesellschaftspolitischen Perspektivwechsel}

Aktivitäten und Arbeiten, die eine postmigrantische Sicht auf Gesellschaft proklamieren, richten sich nicht nur auf die analytische Erfassung und Anerkennung von ,Diversity“. Sie fordern vielmehr dazu auf, ,den hegemonialen Migrationsdiskurs aus der Perspektive und Erfahrung von Migration zu de- und rekonstruieren“" (Y1ld1z, 2016:42). Kennzeichnend ist somit neben einer gesellschaftsanalytischen Neuausrichtung besonders die kritisch-normative Perspektive. Diese Orientierung steht im engen Zusammenhang mit der kulturellen und politischen Kontextualisierung des Begriffs „Postmigration“ im deutschsprachigen Raum, insbesondere hinsichtlich der Geschichte der Zuwanderung im Zuge der ab 1955 geschlossenen Anwerbeabkommen. So wurde der Begriff in der Bundesrepublik zunächst von Künstlern und Aktivistinnen aufgegriffen, die auf der Grundlage familiärer oder persönlicher Migrationserfahrungen auf eine stärkere gesellschaftliche Anerkennung von Einwanderungsgeschichte abzielten und zu einer Sensibilisierung für Diskriminierungen beitragen wollten (u. a. Langhoff, 2012; Espahangizi, 2016; Y1ldız, 2015; Hill und Y1ldız, 2018). Ein zentrales Anliegen war es, das Bewusstsein für die gesellschaftliche Bedeutung von individuell-subjektiven Migrationserfahrungen auch gezielt in aktuelle stadtpolitische Debatten einzubringen. Das „Postmigrantische“ wurde so auch zu einer Art Kampfbegriff, der sich explizit gegen eine Marginalisierung von Menschen mit Migrationsbiografie richtet und der einen gesellschaftlichen Diskurs kritisiert, der zwischen einer unterstellten mehrheitsgesellschaftlichen Normalität und durch Zuwanderung verursachten Problemen unterscheidet (Y1ldiz, 2015:22). Der Begriff ist somit zunächst vor allem als eine Art Appell zu verstehen, Gesellschaft anders und zwar vom Standpunkt der Migrationserfahrung heraus neu zu denken und dadurch das Bewusstsein für natio-ethno-kulturelle Differenzmarkierungen zu schärfen. Ein wesentlicher Aspekt besteht nicht zuletzt in der Formulierung einer ,neuen Rassismuskritik", die sich auf einflussreiche gesellschaftliche Diskurse und Praktiken richtet, die über die Reproduktion von Rasse- oder Kulturkonstruktionen gesellschaftliche Ungleichbehandlungen erst wirksam machen (Espahangizi et al., 2016:15; Castro-Varela und Mecheril, 2016). Fest steht, dass sich der Zugang durch ein besonderes Potenzial auszeichnet, den konventionellen Blick auf migrationsbedingte Veranderungen und Ausgrenzungen gegen den Strich zu bürsten, das gesellschaftliche „Hadern“ mit der Migration (Spielhaus, 2014:97) in den Blick zu nehmen und die Relevanz eines gesellschaftlichen Perspektivwechsels aufzuzeigen. Der Mehrwert eines postmigrantischen Zugangs wird damit neben dem kritischen Blick auf die gesellschaftliche Konstruktion nationaler Grenzen und Zugehörigkeiten vor allem im Aufzeigen von persistenten Machtkonstellationen sowie in der Dekonstruktion hegemonialer Kategorien gesehen - ein Anliegen, das er jedoch mit anderen Ansätzen teilt.

\subsection{Das „Postmigrantische“ zwischen gesellschaftstheoretischem Anspruch und empirischen Herausforderungen}

Inwieweit eine ,postmigrantische Perspektive“ auf Gesellschaft auch als ein wissenschaftlich tragfähiges Konzept in Wert gesetzt werden kann, wird kontrovers diskutiert (u. a. Mecheril, 2014). Irritationen provoziert hier insbesondere die Vorsilbe ,post-“, die oft fälschlicherweise in einem chronologisch-linearen Sinn interpretiert wird. $\mathrm{Zu}$ verstehen ist sie jedoch im Sinn eines Aufbrechens jener gewohnten Denkmuster, die mit den Debatten um Zuwanderung verknüpft sind (u. a. Langhoff, 2012; Hill und Y1ldı, 2018:7). Im Zentrum des Ansatzes steht die Forderung, die konventionelle Migrationsforschung aus ihrer Sonderrolle $\mathrm{zu}$ befreien und durch eine Gesellschaftsanalyse zu ersetzen, „,die alle zu ,Betroffenen“ der Migration und zu GestalterInnen der dadurch konstituierten Verhältnisse erklärt" (Bojadžijev und Römhild, 2014:18f; Y1ldız, 2015:22). Eine entsprechend konsequente Anerkennung migrationsgesellschaftlicher Realitäten setzt jedoch auch adäquate analytisch-konzeptionelle Zugänge voraus. Denn wenn die Prämisse gilt, ,dass Migration als ein normaler Bestandteil der Gesellschaft anzusehen ist, das heißt, alle Menschen in irgendeiner Form mobil sind und in einer Migrationsgesellschaft leben, dann muss Migrationsforschung sich mit der Analyse der gesamten Gesellschaft beschäftigen“ (Hill, 2018:100/101). Noch unzureichend geklärt bleibt dennoch, wie empirische Forschung angelegt sein muss, um Migrationsgesellschaften jenseits von kulturalistisch-ethnisch-nationalen Zuschreibungen und Kategorisierungen als Ganzes in den Blick zu nehmen. Denn das Ziel, Lebenswirklichkeiten aus der Perspektive der Migration zu erforschen - „Gesellschaftswissenschaften zu migrantisieren“ (Bojadžijev und Römhild, 2014:11; 20f) -, beinhaltet naturgemäß den Bezug auf gesellschaftliche Differenzierungs- und Kategorisierungssysteme. Damit kann gerade das kritisch-normative Moment der postmigrantischen Perspektive, welches darauf abzielt, die Diskriminierung und ,Migrantisierung“ von Bevölkerungsgruppen kritisch in den Blick zu nehmen, zu einer Reproduktion kategorisierender Ein- und Ausschlüsse beitragen. So kommt auch der kritisch-postmigrantische Ansatz oft nicht umhin, „Migrationsandere“ als solche zu markieren. Dadurch läuft er Gefahr, in Dichotomien wie ,Migrant/Nicht-Migrant“, ,einheimisch/nichteinheimisch“, „hegemonial/gegenhegemonial“ verhaftet $\mathrm{zu}$ bleiben (z. B. Dahinden, 2016; Römhild, 2015 sowie West in diesem Heft). Auf der anderen Seite können die Bemühungen, natio-ethno-kulturelle Differenzmarkierungen zu überwinden, auch zu Einschränkungen in den analytischen oder thematischen Zugängen führen oder dazu, Forschungsfragen, 
die im Kontext von Migrationserfahrung und Herkunft stehen, zu dethematisieren. Auch das explizit formulierte Anliegen eines postmigrantischen Perspektivwechsels, Migration vom Rand in das Zentrum der Gesellschaft zu bringen (Labor Migration, 2014), kann Gefahr laufen, dahingehend missverstanden zu werden, nur jene in den Blick zu nehmen, die sozial und beruflich in der Mitte der Gesellschaft angekommen sind und subalterne Milieus von der Betrachtung auszuschließen ${ }^{1}$. Während die vielfältigen Studien, die sich unter dem Dach des Begriffs „Diversity“ subsumieren lassen, eher in der Tradition stehen, hochkomplexe Variablensets zu beschreiben und auf einen entpolitisierten Vielfaltsbegriff abzuheben, stehen im gesellschaftskritisch ausgerichteten postmigrantischen Zugang vor allem noch Strategien aus, die geeignet sind, die neuen gesellschaftlichen Verhältnisse empirisch abzubilden, ohne selbst in die Falle von Veranderungen und kulturalistischen Reduktionen zu tappen (siehe auch Römhild, 2015:38). Die Herausforderung besteht nicht zuletzt darin, einerseits die Unterscheidung ethnisierender oder kulturalisierender Kategorisierungen zu überwinden und andererseits dennoch Migrationserfahrungen mit ihren vielfältigen Ursachen sowie den damit verbundenen Hürden und Ausschlüssen im Blick zu behalten (Çağlar und GlickSchiller, 2018:5). Gayatri Chakravorty Spivaks Konzept des „strategischen Essentialismus“, das eine taktische Verwendung von essentialistischen Identitäten im Rahmen von klar definierten politischen Zielen verficht, kann eine Möglichkeit darstellen, mit diesem grundlegenden Konflikt produktiv umzugehen (Spivak, 1988; siehe auch Dahinden, 2016).

Unter einer wissenschaftlich-analytischen Zielsetzung lässt sich das „Postmigrantische“ vor allem als eine Analysekategorie für soziale Situationen heranziehen, die von Mobilität und Diversität, aber auch von sozialen Brüchen, Grenzbiografien und Mehrdeutigkeiten sowie von Prozessen der Entortung und Neuverortung geprägt sind (Y1ld1z, 2015:22). Damit kann sich der Blick bevorzugt auf gesellschaftliche Praktiken und Diskurse richten sowie auf subjektive Positionierungen in der gesellschaftlichen Auseinandersetzung mit Heterogenität und Pluralität (z. B. Berding in diesem Heft). Ein weiteres wesentliches Moment ist es zu erforschen, ,wie ethnisierte und ,migrationalisierte "Weltansichten - von Individuen, in Institutionen und Politik etc. - hervorgebracht werden, sich verändern und mit anderen Perspektiven über ,Differenz“ interagieren“ (Dahinden, 2016:18). Damit steht die Dekonstruktion der sozialen Produktion von ,Migration" als Ergebnis historischer und raumbezogener Kategorisierungen und Narrative der Zugehörigkeit bzw. Nicht-

\footnotetext{
${ }^{1}$ In diesem Zusammenhang fragt z.B. Supik mit Bezug auf Stuart Hall auch kritisch: „,.. ob durch das Eintreten Marginalisierter in Bereiche des Zentrums ein Erfolg für diese zu verbuchen ist oder dies als Vereinnahmung betrachtet werden muss. Wo fängt Marktgängigkeit an und wo hört Anerkennung auf? Wo schlägt die eine in die andere um? Wann erweist sich Hybridität als subversives Potential, und wo ist sie nur der letzte Schrei nach Unterhaltung?" (Supik, 2005:114).
}

Zugehörigkeit im Zentrum, auf deren Grundlage unter anderem kontextabhängige Grenzen zwischen „MigrantInnen“ und „Nicht-MigrantInnen“ gezogen werden (z. B. auch in Form eines „Doing Migration Approaches“ bei Anna Amelina, 2017). Von besonderer Erklärungskraft sind in diesem Zusammenhang lokale Entwicklungspfade, Diskursprägungen, Institutionen sowie spezifische Akteurs- und Machtkonstellationen, die darauf einwirken, wie migrationsbedingte Ein- und Ausschlüsse jeweils ausgehandelt und gestaltet werden (Pott, 2018:121). Vor diesem Hintergrund sollen entsprechend der kritisch-normativen Ausrichtung des ,Postmigrantischen“ im Folgenden Anschlussmöglichkeiten mit Konzepten der kritischen Stadtforschung im Zentrum stehen.

\section{Postmigration und Stadtforschung}

Dass sich migrationsbezogene Aushandlungen trotz scheinbar zunehmender gesellschaftlicher Raumunabhängigkeit immer in spezifischen raumbezogenen Kontexten vollziehen, liegt auf der Hand. Lebensstile und Kulturen, die oft weit voneinander entfernt praktiziert werden, werden auf lokaler Ebene neu kombiniert und führen $\mathrm{zu}$ neuen Formen lokaler Verankerungen. Im Umfeld der Diversity Studies ist mit der expliziten Hinwendung zu Spaces of Diversity eine deutliche Orientierung auf konkrete lokale Verankerungen und die Analyse lokalisierter Formen von Diversität erkennbar (z. B. Berg und Sigona, 2013:3). Im Umfeld postmigrantischer Zugänge sind räumliche Bezüge dagegen im Allgemeinen noch wenig explizit thematisiert worden, zum Teil bleiben sie auch überraschend klassisch auf Quartiere mit hohen Anteilen an Bewohnerinnen und Bewohnern mit Migrationshintergrund fokussiert (z. B. Hill, 2016). Der Eindruck, dass insbesondere der Zusammenhang von Stadt und Migration in migrationswissenschaftlichen Arbeiten untertheoretisiert bleibt, wird u. a. darauf zurückgeführt, dass der Bezug auf „Stadt“ oft als implizit gesetzt und selbstverständlich gegeben angenommen wird (Hess und Lebuhn, 2014b:6). Legt man die Durchgängigkeit und Multidirektionalität von Migrationserfahrungen auf unterschiedlichen Bezugsebenen als wesentliches Element des postmigrantischen Gedankens zugrunde, bildet ein relationales Raumverständnis eine wesentliche konzeptionelle Voraussetzung für eine postmigrantisch informierte Stadtforschung. Dieses ermöglicht es, ,räumliche Ent- und Wiederverankerungspraxen sowie die soziale Einbettung von Migration in multilokale Zusammenhänge und die Herstellung der dafür erforderlichen Ortsbezüge“" analytisch zu fassen (Pott, 2018:115). Besonders im Umfeld der Transnationalismusforschung mit ihrem Fokus auf globale Machtbeziehungen und der Untersuchung translokaler und transnationaler Sozialräume bzw. Netzwerke finden sich Beispiele für entsprechende analytische Zugänge (z. B. Çağlar und Glick-Schiller, 2018). Postmigrantische Studien greifen diese Gedanken mit dem Begriff der Transtopien auf, ,in denen scheinbar entfernte, lokale wie grenzüberschreitende 
Elemente miteinander verknüpft werden und sich zu urbanen Strukturen und Kommunikationsformen verdichten" (Yıldız, 2015:32). Hinsichtlich eines Zusammendenkens von Stadtforschung und einer postmigrantischen Perspektive auf Gesellschaft wird besonders auch die grundlegende theoretischkonzeptionelle Relevanz der Arbeiten deutlich, die sich auf Migrationsregime beziehen (Pott et al., 2018). Trotz der unterschiedlichen Interpretationen und disziplinären Anwendungen der Regimeperspektive zeigt sie in ihrer Zielsetzung, „die Komplexität der Aushandlungsprozesse von Migration unter einem Dach auszuloten“ (Pott et al., 2018:11), viele Anknüpfungspunkte. So stellt sowohl der postmigrantisch orientierte Zugang als auch der Ansatz des Migrationsregimes die Distanzierung und Dekonstruktion von überkommenen Machtverhältnissen sowie die ,reflexive(r) Besinnung der Migrationsforschung über ihre Konzepte, Begriffe und letztlich ihre eigene Position“ (Pott et al., 2018:12; siehe auch Horvath et al., 2017:310f) zentral. Hinsichtlich seines spezifischen Entstehungskontexts lässt sich ,das Postmigrantische“ selbst als ein spezifischer Akteurs-DiskursPraxis-Zusammenhang im Sinn des Regimeansatzes interpretieren: als Ausdruck komplexer, multiskalarer Aushandlungen um Migration durch unterschiedliche Akteure und Machtverhältnisse im raumzeitlichen Kontext der westdeutschen „Gastarbeiterzuwanderung“ und migrationsbezogener Kämpfe um Anerkennung in Großstädten der Bundesrepublik Deutschland. Ein zentrales Moment richtet sich hier auf die normativ-emanzipatorische Frage, wer zur Stadtgesellschaft gehört und wer nicht, wer wann aus welchen Systemen ausgeschlossen wurde und welchen Personengruppen welche Formen der sozialen Teilhabe zugesprochen werden. Mit der Forderung nach gleichberechtigter Teilhabe und sozialer Partizipation - unabhängig von Herkunft und nationalen Normen - weist das „Postmigrantische“ wiederum zahlreiche Anknüpfungspunkte zur Debatte um das Recht auf Stadt und „Urban Citizenship“ auf. Letztere richtet sich einerseits auf ,,die Perspektive des städtischen Regierens der Migration" und ermöglicht es andererseits, die migrationsbezogene Agency ,,jenseits ethnisierender und kulturalisierender Paradigmen zu thematisieren“ (Hess und Lebuhn, 2014a:13).

\section{1 „Urban Citizenship“ und postmigrantische Kämpfe um das Recht auf Stadt}

Die Frage nach lokalen Praktiken und Politiken im Umgang mit Migration richtet sich nicht zuletzt auf alle Aspekte der Teilhabe in unterschiedlichen gesellschaftlichen Subsystemen wie insbesondere den Wohn- und Arbeitsmärkten, den Bildungssystemen sowie den Möglichkeiten der politischen Partizipation. Der Bezug auf Lefebvres „Recht auf Stadt“ (1986) im Sinn eines „Rechts auf Nichtausschluss“ von den Leistungen und Qualitäten der städtischen Gesellschaft (Holm, 2011) kann diesbezüglich einen Ausgangspunkt bilden, um die Fragen nach Zugehörigkeiten zur Stadtgesellschaft aus einer postmigrantischen Perspektive zu be- leuchten. In diesem Zusammenhang stehen nicht zuletzt Forderungen nach einem Umdenken ,des Urbanen“ im Sinn einer Entmarginalisierung von (post-)migrantischen Lebenskonzepten sowie der Anerkennung der Beiträge von Migrantinnen und Migranten zur Stadtentwicklung und Urbanisierung. Politisch orientiert sich das Recht auf Stadt an der Ermöglichung einer demokratischen Mitgestaltung für alle (Holm, 2011). Dabei geht es aber weniger um Repräsentanzen in einem multikulturalistischen Sinn, sondern vielmehr darum, im alltäglichen institutionellen Handeln der steigenden Diversität und Pluralität der Gesellschaft generell gerecht zu werden. Nicht zuletzt sich als postmigrantisch verstehende Aktivistinnen und Aktivisten zielen in diesem $\mathrm{Zu}$ sammenhang auf eine größere öffentliche Aufmerksamkeit sowie Formen der gesellschaftlichen und politischen Anerkennung jenseits von nationalstaatlichen Formen der Zughörigkeit ab (Ataç et al., 2015). In einem übergeordneten Sinn richten sich entsprechende Betrachtungen des Urbanen auf Fragen nach globalen Menschen- und Bürgerrechten, die all diejenigen einbeziehen, die in den Städten leben wollen unabhängig von ihrem Aufenthaltsstatus (siehe z. B. West in diesem Heft). Ein wesentliches Moment ist daher das Hinterfragen von nationalstaatlichen Logiken, die einen gesellschaftlichen Ausschluss von Nicht-StaatsbürgerInnen zur Folge haben und dadurch Ungleichheiten institutionalisieren. Mehrebenenmodelle, die zwischen unterschiedlichen Formen der Bürgerschaft unterscheiden, sowie die Idee der „Urban Citizenship“, die auf dem Domizilrecht bzw. auf Anwohnerschaft gründet und nicht auf nationaler Zugehörigkeit, repräsentieren in diesem Zusammenhang vieldiskutierte Konstrukte (z. B. Bauder, 2016:255; Rodatz, 2014; Bauböck, 2003).

Aktuelle Beispiele, die an den Gedanken einer „Urban Citizenship“ anschließen, sind kommunale Bewegungen der Solidarität, die sich in der Bundesrepublik vor allem im Kontext des langen Sommers der Migration im Jahr 2015 entwickelt haben. So haben sich unter den Stichworten „Sanctuary City“, Städte der Zuflucht oder „Solidarity City“ neue Zusammenschlüsse von zivilen und staatlichen Akteuren formiert, mit dem Ziel, Städte zu einem ,sicheren Hafen“ für Menschen ohne Aufenthaltsstatus zu erklären und Abschiebungen $\mathrm{zu}$ verhindern. Eine postmigrantisch orientierte Stadtforschung kann hier einen thematischen Fokus auf Formen von Urbanität richten, die über die Konstitution von neuen gesellschaftlichen und politischen Allianzen erzeugt werden. Durch entsprechende Bündnisse auf unterschiedlichen Ebenen, die Vertreter kommunaler Verwaltungen, Bürgerinitiativen ebenso einschließen können wie ,,illegalisierte" Personen, können soziale Grenzen und Hierarchien zwischen unterschiedlichen Akteurinnen und Akteuren in der Stadt phasenweise aufgehoben werden (Bauder, 2016). Die Konflikte um migrationsbezogene Teilhabe und Partizipation sowie die mikropolitischen Formen des Widerstandes im Feld der Debatte um das Recht auf Stadt sind meist an konkrete Orte geknüpft. Gleichzeitig ist ihre Einbettung in welt- 
weite, transnationale Konstellationen und Mobilitätszwänge zentral. Ansätze im Umfeld der „Postcolonial Urban Studies" fordern diesbezüglich zu einem kritischen Blickwechsel auf urbane Aushandlungen vor Ort und auf Bezüge zwischen urbanen Konflikten an ganz unterschiedlichen Orten der Welt heraus (siehe z. B. auch Lanz, 2015).

\subsection{Postmigrantische Stadt - ein postkolonial orientierter Analysefokus für städtische Zuwanderungsgesellschaften?}

Die zunehmende Bedeutung transkultureller Bezüge und sozioökonomischer Verflechtungen hat nicht nur den Blick auf Stadtgesellschaften verändert, sondern auch dazu beigetragen, die Vormachtstellung westlicher Theoriebildung in der Stadtforschung kritisch infrage zu stellen. Vor allem im Umfeld der „Postcolonial Urban Studies“ wird die Existenz einer einzigen übergeordneten theoretischen Erzählung der Urbanisierung hinterfragt und mit einer selbstkritischen Reflexion des ,westlichen“ Forscherblicks konfrontiert (z. B. Lanz, 2015; Robinson, 2006). Im Mittelpunkt steht ein kritisches Bewusstsein für die asymmetrischen Machtverhältnisse zwischen dem „Wir" eines fortschrittlichen Westens und den „Anderen“ bzw. „dem Rest“ (Hall, 1994) in der Stadtforschung. So werden wie im Ansatz der „Ordinary Cities“ (Robinson, 2006) Perspektiven für transnationale Vergleiche eröffnet, die darauf abzielen, ausgrenzende Kategorisierungen oder Hierarchisierung von Städten zu hinterfragen und zu überwinden (u. a. McFarlane und Robinson, 2013). Etablierte Konzepte wie das der europäischen Stadt können durch entsprechende Perspektivwechsel als eine Diskursformation dekonstruiert werden, die „das Westliche als städtisch, modern, zivilisiert und säkularisiert, den ,Rest" hingegen als unterentwickelt, traditionell und religiös deutet" (Lanz, 2015:76; siehe auch Lossau, 2012 126; Ha, 2014). Diese Stoßrichtung kann die vielen grundlegenden Parallelen zwischen postkolonial orientierter Stadtforschung und einer postmigrantischen Perspektive auf Gesellschaft an dieser Stelle nur andeuten (siehe u. a. Yıldız, 2015; Liebig, 2015:7). So unter anderem in der Kritik an linearen Entwicklungsmodellen wie der postmigrantischen Kritik am Integrationsparadigma sowie analog dazu der Kritik der „Postcolonial Studies" am Modernisierungsparadigma. Ebenso wie der postmigrantische Zugang eine Defizitperspektive auf Migration kritisiert, basiert ein Kerngedanke der „Ordinary Cities“ auf der Kritik an der Vorherrschaft einer einzigen, westlich geprägten theoretischen Erzählung der Urbanisierung, die impliziert, andere urbane Entwicklungen weltweit aus einer Defizitperspektive heraus zu betrachten. Postkoloniale Stadtforschung zielt demgegenüber auf die Auseinandersetzung mit der kulturellen Vielfältigkeit des Städtischen ab, ohne von einer universellen Anwendbarkeit urbaner Konzepte - insbesondere euroamerikanischer Perspektiven und westlich zentrierter Theoriebildung - auszugehen oder auf eine Kategorisierung der Vielfältigkeit städtischer Entwicklungen abzu- heben (Robinson, 2006:60). Sowohl postkoloniale Perspektiven auf Stadt als auch postmigrantische Sichten auf Gesellschaft stellen dabei die Bedeutung und die Wechselbeziehungen zwischen historischen und gegenwärtigen Machtverhältnissen zentral. Ähnlich wie im Umfeld postmigrantischer Zugänge ist in der vergleichenden Stadtforschung unter dem Stichwort „Transfer“ das Interesse an wechselseitigen Beziehungen und Einflüssen zwischen unterschiedlichen städtischen Kulturen in den Vordergrund gerückt (Robinson, 2006). Dementsprechend fordert eine postmigrantische Perspektive auf Stadt u. a. ein stärkeres Bewusstsein für die historischen Verwobenheiten von Stadt- und Migrationsgeschichte, wie u. a. die Frage, welche Erinnerungspraktiken, die sich auf Wanderungsbewegungen beziehen, Bestandteile des städtischen Alltags bilden (z. B. Hess und Näser, 2015). Postmigrantische Stadtforschung lässt sich so als eine Spielart postkolonialer Stadtforschung interpretieren: Eine Stadtforschung mit einem besonderen Fokus auf die urbane $\mathrm{Zu}$ wanderungsgeschichte der vergangenen Jahrzehnte und auf Stadtgesellschaften, in denen das Bewusstsein, Einwanderungsgesellschaft zu sein, ein wesentliches Element des gesellschaftspolitischen Diskurses geworden ist ${ }^{2}$. Die Dezentrierung des „westlichen Blicks“ (Hall, 1997 nach Supik, 2005:25) wäre in diesem Zusammenhang sowohl auf die Betrachtung der urbanen Gesellschaften selbst als auch auf die gängigen westlich-eurozentrisch geprägten Konzepte in der Stadtforschung anzuwenden. Entsprechende Blickverschiebungen werden auch in den Beiträgen der vorliegenden Special Edition Social Geography getestet.

\section{Die postmigrantische Stadt als Beobachtungsperspektive der sozialgeographischen Stadtforschung - Ausblick auf die Beiträge}

Die Beiträge dieses Themenheftes stellen verschiedene $\mathrm{Zu}$ gänge zur Diskussion, um die beschriebenen reflexiven Wendungen im Schnittfeld von Stadt- und Migrationsforschung konzeptionell zu fassen. Im Mittelpunkt steht die Auseinandersetzung mit der Frage, welcher Mehrwert von einem Perspektivwechsel ausgehen kann, der Migration zum einen nicht als Sonder- oder Problemfall, sondern als gesellschaftliche Normalität begreift, zum anderen aber auch der Bedeutung und dem Einfluss individueller kultureller Bindungen und Identitäten jenseits essentialisierender Kategorisierung Rechnung trägt. In allen Beiträgen des Themenheftes dient der Begriff des Postmigrantischen daher zunächst als ein Set kritischer Fragen, die an das empirische Material gestellt werden. Als thematische Felder im Schnittfeld von Migrations- und Stadtforschung stehen Perspekti-

\footnotetext{
${ }^{2}$ Laut Foroutan kann eine Gesellschaft dann als postmigrantisch bezeichnet werden, wenn das Bewusstsein, Einwanderungsgesellschaft zu sein, politisch anerkannt und Teil des gesellschaftlichen Narrativs geworden ist (Foroutan, 2015:2).
} 
ven auf ,Ankunftsquartiere“ (Berding), auf den kommunalpolitischen Umgang mit sogenannten „,migrantischen Ökonomien" (Räuchle und Nuissl) sowie auf Prozesse des Ankommens und des Einlebens von Menschen mit Fluchterfahrungen in unterschiedlichen urbanen Kontexten (Weiss et al.) im Zentrum. Die Beiträge stellen damit die Frage in den Mittelpunkt, welche Konsequenzen es für eine empirische und angewandte Forschung hat, wenn eine ,postmigrantische Perspektive" auf klassisch stadtgeographische Fragestellungen angewendet wird. Ein wesentlicher Aspekt ist hier die kritische Auseinandersetzung mit defizit- als auch potenzialorientierten Perspektiven auf Migration sowie ein problematisierender Umgang mit Integrationsbegriffen. Eine ressourcenorientierte, ökonomische Bewertung von Zuwanderung bis hin zu ihrer Instrumentalisierung stellt nach wie vor einen dominanten Diskursstrang gegenwärtiger Stadtentwicklungspolitiken dar. Migration und Vielfalt werden so als wichtige Faktoren im zwischenstädtischen Wettbewerb um Unternehmen und Fachkräfte gehandelt. Auch die Inszenierung von Diversität im Kontext von Aufwertungsprozessen ist eine Facette des Potenzialdiskurses um Migration im Zeichen neoliberaler Stadtentwicklungspolitiken (z. B. Rodatz, 2014; Pütz und Rodatz, 2013; Lanz, 2007). Gleichzeitig kommen jedoch - zum Teil durchaus mit der Aufwertung von Stadträumen in Zusammenhang stehend - weiterhin ausschließende Praktiken im Umgang mit internationaler Zuwanderung zum Tragen (z. B. Tsianos, 2014; Wiest und Kirndörfer, 2019). Das Spannungsverhältnis von potenzialund problemorientierten Diskursen dominiert insbesondere Studien, die die Ebene von städtischen Nachbarschaften in den Blick nehmen. So haben sich in den letzten Jahren zahlreiche theoretische und empirische Arbeiten mit Fragen des sozialen Zusammenhalts und des Zusammenlebens auf der Quartiersebene auseinandergesetzt. Im Fokus stehen hier besonders Nachbarschaften in Großstädten, die im starken Maß durch soziale und natio-ethno-kulturelle Diversität geprägt sind. Diskutiert werden einerseits sich verstärkende räumliche, soziale und symbolische Grenzziehungen entlang natioethno-kultureller Zuschreibungen (Albeda et al., 2018) bis hin zu Debatten um Parallelgesellschaften (z. B. kritisch dazu Tsianos und Ronneberger, 2012). Zum anderen wurden vor allem unter dem Label „Ankunftsquartier“ die integrationspolitischen Potenziale und Chancen von superdiversen Stadträumen ausgelotet (Saunders, 2010; Hans et al., 2019).

\subsection{Postmigrantische Alltagsroutinen, „Normalcy of Diversity“ und Verschiebungen von Normalität}

In deutlicher Abgrenzung zu problem- und potenzialorientierten Perspektiven auf diversitätsgeprägte Quartiere erforscht Nina Berding in ihrem Beitrag das alltägliche $\mathrm{Zu}-$ sammenleben im Düsseldorfer Stadtteil Oberbilk. Nicht zuletzt, um im Sinn einer postmigrantischen (Stadt-)Forschung einer ,migrantisierenden“ Sicht auf das urbane Zusammenleben zu entgehen, stellt Berding jene „Vielverarbeitungs- strategien" und routinierten Praktiken in das Zentrum, die Stadtbewohner und Stadtbewohnerinnen entwickeln, um sich in einem komplexen städtischen Alltag zurechtzufinden. Über den Rückgriff auf Simmels Begriff der „Blasiertheit“ im Sinn eines inklusiven Verhaltensstils unter den Bedingungen von Komplexität wird Diversität zu einer gelebten Selbstverständlichkeit, die sich in den pragmatischen Haltungen, Handlungsmustern und sozialen Beziehungen der Stadtgesellschaft widerspiegelt. Der Mehrwert einer postmigrantischen Perspektive wird in diesem Untersuchungszusammenhang dahingehend interpretiert, dass die Erfahrung von Diversität als eine gemeinsame und selbstverständliche Handlungs- und Wahrnehmungsgrundlage thematisiert wird. Dadurch rücken die StadtbewohnerInnen selbst als distanziert-konstruktiv handelnde AkteurInnen in den Mittelpunkt. Während Berding Aspekte einer Alltäglichkeit, einer ,Normalcy“ bzw. ,Everydayness of Diversity“ (Wessendorf, 2014:2; Meissner, 2015:557) im Sinn von Konvivialität und pragmatischer Routine zentral stellt, stellt sich gleichzeitig die Frage, inwieweit und in welchen Kontexten es dagegen zu Verschiebungen von entsprechenden Normalitäten kommt und ein Aufbrechen von Routinen in Gang gesetzt wird, das neue, emotionalisierte Ausgrenzungen jenseits von Blasiertheit im Sinn pragmatischer Distanz produziert. Beispiele dafür finden sich $u$. a. in Untersuchungen zur Ausgrenzung von Geflüchteten und in Befunden zum Entstehen feindseliger Orte (z. B. Kurtenbach, 2019). Damit soll vor allem auf die widersprüchlichen diskursiven Dynamiken hingewiesen werden, die charakteristisch für hochmobile und internationalisierte Gesellschaften sind: Während routinierte Distanzierungen und Grenzziehungen wie im Sinn blasierter Haltungen wichtige inklusive Ressourcen repräsentieren können, werden in anderen Kontexten zunehmend desintegrative Effekte gesellschaftlicher Grenzziehungen spürbar, wie nicht zuletzt in Form von neuen Rassismen (Espahangizi et al., 2016:15).

\subsection{Perspektiven auf Integration und Ankommen vom Standpunkt der Migration}

Die aufgrund von Fluchtursachen verstärkte Zuwanderung zwischen 2014 und 2016 hat eine Reihe an Studien hervorgebracht, die sich besonders mit dem Umgang und der Wahrnehmung der Ankunft von Menschen mit Fluchtbiografie in spezifischen lokalen Kontexten der Bundesrepublik auseinandergesetzt haben (u. a. Hamann und Yurdakul, 2018; Glorius et al., 2019; Kurtenbach, 2019). Unter Anerkennung globalisierter Bedingungen und im Sinn eines postmigrantischen Zugangs ist Fluchtmigration jedoch weniger als Sonderfall zu betrachten, sondern als Zustand anzunehmen, der - durch weltweite ökonomische Disparitäten und politische Machtgefälle - immer Bestandteil globaler Mobilitätsprozesse ist. Durch diese Perspektive kann sich auch der Blick auf die gängige Dichotomie zwischen einem „Wir“ der Aufnahmegesellschaft und den „Anderen“ mit Fluchterfahrung 
wandeln. Entsprechende Dichotomien zu hinterfragen und ggf. aufzuheben ist eine der grundlegenden Herausforderungen von Arbeiten, die eine postmigrantische Perspektive auf Fluchtmigration einnehmen (z. B. Ratković, 2017). Dieser Aspekt ist auch ein wichtiges Moment des Beitrags von Günther Weiss, Francesca Adam, Stefanie Föbker, Daniela Imani, Carmella Pfaffenbach und Claus-Christian Wiegandt. Ausgehend von einer Forschungsarbeit über Prozesse des Ankommens und Einlebens von Geflüchteten in zwei westdeutschen Kommunen in Nordrheinwestfalen, versucht das Autorenteam, sich einem Integrationsverständnis zu nähern, das von der subjektiven Sicht der unterschiedlichen Akteurinnen und Akteure ausgeht. Die Sichtweisen von Geflüchteten und von Menschen, die in der Hilfe für Geflüchtete tätig sind, werden im Rahmen dieses Beitrags zwar auch gegenüberstellt. Diese Unterscheidung ist jedoch den grundsätzlich sehr ungleichen gesellschaftlichen (Macht-)Positionen inhärent und wird nicht etwa mit natio-ethno-kulturellen $\mathrm{Zu}$ schreibungen begründet. Der Ansatz zielt hier vielmehr auf eine gleichberechtigte Gegenüberstellung ab, die besonders die Sicht der Gesprächspartnerinnen und Gesprächspartner mit Fluchtbiografie in den Mittelpunkt rückt. Die Sichtweisen und Vorstellungen der beiden Gruppen lassen dabei zum Teil mehr Gemeinsamkeiten als Unterschiede erkennen und machen deutlich, dass „Integration“ hier in der Regel nicht als ein einseitiger Imperativ wahrgenommen wird (siehe z. B. auch Hamann, 2019). Die Einsichten lassen aber auch erahnen, dass die Erfahrung der unmittelbar Betroffenen, sei es der Geflüchteten selbst oder der in der Hilfe für Geflüchteten Tätigen, oft weitgehend abgekoppelt von herrschenden öffentlichen Diskursen verlaufen, in denen Abgrenzungen und Ängste dominieren.

\subsection{Kritische Reflexion von Essentialisierungen und Potenzialdiskursen}

Die Agency von Migrantinnen und Migranten und ihr Einfluss auf Stadtentwicklung ist besonders häufig im Hinblick auf die Bedeutung migrantischer Ökonomien thematisiert worden (Hillmann, 2011). Die Etablierung von migrantischen Unternehmen wird einerseits als Reaktion auf $\mathrm{Zu}$ gangsbarrieren auf den städtischen Arbeitsmärkten im Sinn eines „sozialen Aufstiegs auf eigene Rechnung“ (Yildız, 2015:24) interpretiert. Andererseits findet sie Beachtung als Ausdruck eines selbstständigen, gestalterischen SichEinbringens in die ökonomischen und infrastrukturellen Entwicklungen der Stadt (Hillmann, 2011:16). In diesen Bereich fällt auch die strategische Inszenierung ethnischer Authentizität als einer unternehmerischen Vermarktungsstrategie migrantischer Entrepreneure (Stock, 2013). Aus einer dezidiert postmigrantischen Sicht implizieren Untersuchungen zu migrantischen Ökonomien jedoch häufig eine unkritische gesellschaftliche Veranderung und Sonderstellung des unternehmerischen Handelns von StadtbewohnerInnen mit Migrationserfahrungen sowie eine Nähe zu neolibera- len Instrumentalisierungen von Migration. Ein wesentlicher Punkt ist, dass durch die Etikettierung als ,migrantisches“ Unternehmen zwangsläufig spezifische dichotome Verhältnisse im Sinne von „Wir“ und die „Anderen“ konstruiert werden, die darüber hinaus in keinem direkten Verhältnis zur Diversität der Betriebe und den individuellen Strategien und Praktiken ihrer AkteurInnen stehen. In einer kritischen Auseinandersetzung mit diesen Einschränkungen setzen sich Charlotte Räuchle und Henning Nuissl auf Grundlage ihrer Untersuchungen zu „migrantischen Ökonomien“ in deutschen Kommunen mit der Frage auseinander, inwieweit sich dieser eigentlich dezidiert ,präpostmigrantische“ Zugang dennoch für eine postmigrantisch informierte Forschung fruchtbar machen lässt. Zentrale Anknüpfungspunkte werden in der Hinterfragung des Migrationsbegriffs, der Bezugnahme auf Transnationalität sowie in einer problematisierenden Haltung gegenüber einer ,,neoliberalen Inwertsetzung" von Migration gesehen.

\subsection{Transversale versus postmigrantische Perspektive?}

Im Spannungsfeld von aktiven Zuwanderungs- und reaktiven Integrationspolitiken sind in deutschen Kommunen vielerorts umfangreiche politische, zivilgesellschaftliche und verwaltungstechnische Strukturen und Strategien im Umgang mit Zuwanderung geschaffen worden. Die Frage, inwieweit diese lokalen Strategien unter anderem unter postmigrantischen Vorzeichen neu eingeordnet werden können und inwieweit sich daraus innovative Optionen für Stadtentwicklungspolitiken denken lassen, bildet einen Ausgangspunkt für Christina Wests philosophischen Blick auf Gesellschaft und Stadt. Hier entwirft sie das Modell einer "Transversalen Stadt" im Sinn eines Gedankenspiels, urbane Zukunft sowohl konzeptionell als auch empirisch im diskursiven Feld von Migration und Integration neu zu denken. Durch die Identifizierung von vier charakteristischen diskursiven - nämlich homogenisierenden, kritischen, kosmopolitischen und transversalen - Momenten setzt sie unter anderem transversale und postmigrantische Perspektiven zueinander ins Verhältnis. Nach West ist die transversale Perspektive - im Unterschied zur kritisch-postmigrantischen - für die Konstruktion eines „Anderen“, wie den „Migranten“, aber auch des Hybriden unempfindlich und daher am ehesten geeignet, Differenzierungsprinzipien von Migrations- und Vielfaltskonzepten zu überwinden. Während im kosmopolitischen Moment (z. B. im Feld der „Superdiversity“) moderne Kategorisierungsdifferenzierungen wie Kultur, Ethnizität oder Nationalität weiterhin zur Umsetzung einer abstrakten „Vielfalt“ herangezogen werden, zielt das kritische Moment auf Bekämpfung von Diskriminierungen ab (z. B. im postmigrantischen Zugang), aber erzeugt dadurch letztendlich auch migrationsbezogene Kategorisierungen. Transversaler Urbanismus in diesem Verständnis soll der zunehmenden Komplexität der Gesellschaft und der transversalen Orientierung des Einzelnen Rechnung tragen, als miteinander verbundenen Prozes- 
sen und unterschiedlichen Akteuren, die sich quer zu offiziellen oder dominanten Logiken engagieren. Die Herausforderung des Migrations- und Integrationsdiskurses kann nach West darin bestehen, weiter über eine optimistische Erzählung unserer Zukunftsgesellschaft im Sinn einer Transtopie nachzudenken.

Die Beiträge des Themenheftes nehmen nicht zuletzt konkrete lokale Kontexte in den Blick, wie größere Agglomerationsräume (Köln, Rhein-Neckar, Heidelberg), Nachbarschaften im großstädtischen Umfeld wie Düsseldorf-Oberbilk, aber auch agglomerationsfernere urbane Zentren wie Braunschweig, Rostock und Heinsberg. Dadurch wird ein Spektrum an unterschiedlichen stadträumlichen Zusammenhängen abgebildet, in denen Migration und Diversität ausgehandelt werden. Gemeinsam ist den Fallbeispielen jedoch, dass sie sich auf westdeutsche urbane Kontexte beziehen. Diese Verortungen reflektieren in gewisser Weise die Entstehungsgeschichte der postmigrantischen Perspektive, die vor allem in der westdeutschen Migrationsgeschichte seit den 1960er-Jahren begründet liegt. Vor diesem Hintergrund steht nicht zuletzt im deutschsprachigen Raum eine Übertragung des postmigrantischen Blickwechsels auf andere strukturelle Kontexte, wie ländliche oder periphere Räume und Untersuchungszusammenhänge besonders in ostdeutschen Kontexten mit anderen Migrationshistorien, vielfach noch aus. Darüber hinaus stellt sich die Frage, inwieweit sich der Terminus „Postmigration“ auch außerhalb des deutschsprachigen Kontextes als ein tragfähiger erweist. Dies umso mehr, wenn man sich den unterschiedlichen Stand in den Bekenntnissen zu Einwanderungsgesellschaften und im jeweiligen Selbstverständnis, in einer globalen Migrationsgesellschaft zu leben, weltweit und in unterschiedlichen nationalen und städtischen Zusammenhängen vor Augen hält.

Die Beiträge dieses Themenheftes, die klassische stadtgeographische Fragestellungen neu diskutieren, machen darüber hinaus deutlich, dass das Einnehmen einer postmigrantischen Perspektive gerade in der angewandten Stadtforschung viel Potenzial bietet, vorherrschende Denkmuster konstruktiv in Bewegung zu bringen. Dabei scheint es sich besonders in diesen anwendungsbezogenen Feldern als hilfreich zu erweisen, dass das Postmigrantische eben gerade „kein Kind der Akademie“ ist (Espahangizi, 2016). Denn nicht zuletzt, weil der Begriff analytisch (noch) unscharf ist, provoziert er Fragen, regt zum Nachdenken an, zwingt zum Blickwechsel und gibt damit wichtige Impulse - sowohl für die theoretisch-konzeptionelle Auseinandersetzung, aber insbesondere für kritische Selbstreflexionen in der angewandten Stadtforschung wie auch in der kommunalen Praxis. Um sich nicht als Begriff zu überleben, sondern auch nachhaltige gesellschaftliche Debatten anzustoßen, muss der Gedanke des Postmigrantischen aus seiner bislang zum Teil noch avantgardistisch anmutenden Position stärker in breitere urbane Alltagswelten gebracht werden. Dazu wird es notwendig sein, eine unterschiedliche Bildungsschichten, Milieus und Herkunftsgruppen gleichermaßen ansprechende Sprache zu finden, „Übersetzungsmöglichkeiten“ zu bieten, die die Augen für geteilte Geschichten, gemeinsame Erfahrungen und Bedürfnisse als (Stadt-)Bürger und -bürgerinnen trotz unterschiedlichster Sozialisationen öffnen können.

Interessenkonflikt. The authors declare that they have no conflict of interest.

Danksagung. Das vorliegende Themenheft wurde im Rahmen des DFG-Projektes ,Lokal gestrandet, global vernetzt? Umgang mit Vielfalt an den gesellschaftlichen Rändern der postmigrantischen Stadt“ am Leibniz-Institut für Länderkunde (IfL) erarbeitet. Die einzelnen Beiträge wurden in der Fachsitzung „Postmigrantische Stadt? Stadtforschung und der reflexive Turn in der Migrationsforschung“ auf dem Deutschen Kongress für Geographie 2017 präsentiert und diskutiert. Besonderer Dank geht an Elisabeth Kirndörfer und Madlen Pilz für intensive Diskussionen und kritische Hinweise.

\section{Literatur}

Albeda, Y. Oosterlynck, S., und Verschraegen, G.: Symbolic Boundary Making in Super-Diverse Deprived Neighbourhoods, Tijdschrift voor Economische en Sociale Geografie, 109, 470-484, https://doi.org/10.1111/tesg.12297, 2018.

Amelina, A.: After the Reflexive Turn in Migration Studies: Towards the Doing Migration Approach, Working Paper Series „Gender, Diversity and Migration“, Working paper Nr. 13, Goethe University, 2017.

Ataç, I., Kron, S., Schilliger, S., Schwiertz, H., und Stierl, M.: Kämpfe der Migration als Un-/Sichtbare Politik, Einleitung zur zweiten Ausgabe, in: movements, J. Crit. Migrat. Border Regime Stud., 1, 1-18, 2015.

Bauböck, R.: Reinventing Urban Citizenship, Citizenship Stud., 7, 139-160, doi10.1080/1362102032000065946, 2003.

Bauder, H.: Possibilities of urban belonging, Antipode, 48, 252271, https://doi.org/10.1111/anti.12174, 2016.

Berg, M. L. und Sigona, N.: Ethnography, diversitiy and urban space, Identities: Global Stud. Cult. Power, 20, 347-360, https://doi.org/10.1080/1070289X.2013.822382, 2013.

Bojadžijev, M. und Römhild, R.: Was kommt nach dem ,transnational turn"? Perspektiven für eine kritische Migrationsforschung, in: Vom Rand ins Zentrum. Perspektiven einer kritischen Migrationsforschung, Labor Migration, Panama Verlag, Berlin, 10-24, 2014.

Çağlar, A. und Glick-Schiller, N.: Wider die Autonomie der Migration: Eine globale Perspektive auf migrantische Handlungsmacht, in: ZfK - Zeitschrift für Kulturwissenschaften, 2/2011, 147-150, 2011.

Çağlar, A. und Glick-Schiller, N.: Migrants and City-making, Dispossession, Displacement, \& Urban Regeneration, Duke University Press, Durham, London, 2018.

Castro Varela, M. M. und Mecheril, P. (Hrsg.): Die Dämonisierung der Anderen. Einleitende Bemerkungen, in: Die Dämonisierung der Anderen, transcript, Bielefeld, 7-20, https://doi.org/10.14361/9783839436387-001, 2016. 
Dahinden, J.: Migration im Fokus? Plädoyer für eine reflexive Migrationsforschung, in: Migration und Integration - wissenschaftliche Perspektiven aus Österreich, Herausgeber: Schellenbacher, C., Dahlvik, J., Fassmann, H., und Reinprecht, C., V \& R unipress, Göttingen, 11-30, 2016.

El-Mafaalani, A.: Das Integrationsparadox: Warum gelungene Integration zu mehr Konflikten führt, Kiepenheuer/Wisch, Köln, 2018.

Espahangizi, K.: Das \#Postmigrantische ist kein Kind der Akademie, in: Geschichte der Gegenwart, online aufrufbar: https://geschichtedergegenwart.ch/ das-postmigrantische-kein-kind-der-akademie/, letzter $\mathrm{Zu}$ griff: 12 Juni 2016.

Espahangizi, K., Hess, S., Karakayali, J., Kasparek, B., Pagano, S., Rodatz, M., und Tsianos, V.: Rassismus in der postmigrantischen Gesellschaft, Movements, 2, 9-23, 2016.

Foroutan, N.: Die Einheit der Verschiedenen: Integration in der postmigrantischen Gesellschaft, in: focus Migration 28. Kurzdossier, Bundeszentrale für politische Bildung, Bonn, 2015.

Foroutan, N.: Die postmigrantische Perspektive: Aushandlungsprozesse in pluralen Gesellschaften, in: Postmigrantische Versionen, Herausgeber: Hill, M. und Yıldız, E., Transcript, Bielefeld, 1527, 2018.

Glick-Schiller, N., Basch, L., and Blanc-Szanton, C. (Hrsg.): From Immigrant to Transmigrant. Theorizing Transnational Migration, Anthropolog. Quart., 68, 48-63, 1995.

Glick-Schiller, N., Çağlar, A., und Guldbrandsen, T. C.: Beyond the ethnic lens: Locality, globality, and born-again incorporation, Am. Ethnolog., 33, 612-633, 2006.

Glorius, B., Oesch, L., Nienaber, B., and Doomernik, J.: Refugee reception within a common European asylum system: looking at convergences and divergences through a local-to-localcomparison, Erdkunde 73, 19-29, 2019.

Ha, N.: Perspektiven urbaner Dekolonisierung. Die europäische Stadt als „Contact Zone“, Suburban, 2, 27-47, 2014.

Hall, S.: Der Westen und der Rest: Diskurs und Macht, in: Rassismus und kulturelle Identität, Ausgewählte Schriften 2, Herausgeber: Hall, S., Argument, Hamburg, 137-179, 1994.

Hall, S.: Wann war der ,Postkolonialismus'? Denken an der Grenze, in: Hybride Kulturen: Beiträge zur anglo-amerikanischen Multikulturalismusdebatte, Herausgeber: Bronfen, E., Marius, B., und Steffen, T., Stauffenberg Verlag, Tübingen, 219-246, 1997.

Hamann, U.: Eine neue soziale Bewegung? Gespräch mit Ulrike Hamann, in: Die Macht der Migration, Herausgeber: Piening, G., Unrast Verlag, Rosa-Luxemburg Stiftung, Münster, 59-67, 2019.

Hamann, U. und Yurdakul, G.: The transformative forces of Migration: Refugees and the Re-configuration of Migration Societies, Social Inclusion, 6, 110-114, 2018.

Hans, N., Hanhörster, H., Polívka, J., und Beißwenger, S.: Die Rolle von Ankunftsräumen für die Integration Zugewanderter. Eine kritische Diskussion des Forschungsstandes, Raumforschung Raumordnung, 77, 511-524, https://doi.org/10.2478/rara-2019$0019,2019$.

Hess, S. und Lebuhn, H.: Politiken der Bürgerschaft. Zur Forschungsdebatte um Stadt, Migration und citizenship, Suburban, 2, 11-34, 2014a.

Hess, S. und Lebuhn, H.: Editorial: Stadt und Migration, Neue Forschungsansätze zu citizenship, Macht und agency, Suburban, 2, 5-10, 2014b.
Hess, S. und Näser, T. (Hrsg.): Movements of migration. Neue Perspektiven im Feld von Stadt, Migration und Repräsentation, Panama Verlag, Berlin, 2015.

Hill, M.: Nach der Parallelgesellschaft. Neue Perspektiven auf Stadt und Migration, transcript, Bielefeld, 2016.

Hill, M.: Das Stadtleben aus postmigrantischer Perspektive, in: Postmigrantische Visionen. Erfahrungen - Ideen - Reflexionen, Herausgeber: Hill, M. und Yıldı, E., transcript, Bielefeld, 97119, 2018.

Hill, M. und Yıldız, E.: Einleitung, in: Postmigrantische Visionen. Erfahrungen - Ideen - Reflexionen, Herausgeber: Hill, M. und Y1ldı, E., transcript, Bielefeld, 7-19, 2018.

Hillmann, F.: Marginale Urbanität. Migrantisches Unternehmertum und Stadtentwicklung, transcript, Bielefeld, 2011.

Holm, A.: Recht auf Stadt, Blätter für deutsche und internationale Politik, 8/2011, 89-97, 2011.

Horvath, K., Amelina, A., und Peters, K.: Re-thinking the politics of migration. On the uses and challenges of regime perspectives for migration research, Migration Stud., 5, 301-314, https://doi.org/10.1093/migration/mnx055,2017.

Kurtenbach, S.: Kein Platz für Flüchtlinge? Eine empirische Untersuchung der Ausgrenzung Geflüchteter am Beispiel der Stadt Bauten, Raumforschung Raumordnung, 77, 1-16, 2019.

Labor Migration (Hrsg.): Vom Rand ins Zentrum. Perspektiven einer kritischen Migrationsforschung, in: Berliner Blätter, Panama Verlag, Berlin, 2014.

Langhoff, S.: Wozu postmigrantisches Theater?, in: FAZ vom 15 Januar 2012, Interview geführt von Irene Bazinger, online abrufbar: http://www.faz.net/aktuell/feuilleton/buehneund-konzert/gespraechmit-shermin-langhoff-wozupostmigrantisches-theater (letzter Zugriff: 30 Oktober 2019), 2012.

Lanz, S.: Berlin aufgemischt: abendländisch - multikulturell - kosmopolitisch? Die politische Konstruktion einer Einwanderungsstadt, transcript, Bielefeld, 2007.

Lanz, S.: Über (Un-)Möglichkeiten, hiesige Stadtforschung zu postkolonialisieren, Suburban, 3, 75-90, 2015.

Liebig, M.: Das Postmigrantische. Ein neues Konzept für eine kritische Migrationsforschung? Studentische Hausarbeit, veröffentlicht im Labor Migration, online abrufbar: https://www.euroethno.hu-berlin.de/de/forschung/labore/ migration/hausarbeit_postmigrantisch_manuel-liebig.pdf (letzter Zugriff: 30 Oktober 2019), 2015.

Lossau, J.: Postkoloniale Impulse für die deutschsprachige Geographische Entwicklungsforschung, Geogr. Helvet., 67, 125-132, 2012.

McFarlane, C. und Robinson, J.: Introduction - Experiments in Comparative Urbanism, Urban Geogr., 33, 765-773, 2013.

Mecheril, P.: Was ist das X im Postmigrantischen, Suburban, 2, 107-112, 2014.

Mecheril, P.: Ordnung, Krise, Schließung. Anmerkungen zum Begriff Migrationsregime aus zugehörigkeitstheoretischer Perspektive, in: Was ist ein Migrationsregime?, Herausgeber: Pott, A., Rass, C. und Wolff, F., Springer, Wiesbaden, 313-330, 2018.

Meissner, F.: Migration in migration-related diversity? The nexus between superdiversity and migration studies, Ethnic Racial Stud., 38, 556-567, 2015. 
Pott, A.: Migrationsregime und ihre Räume, in: Was ist ein Migrationsregime? What is a Migration Regime?, Herausgeber: Pott, A., Rass, C., und Wolff, F., Springer, Wiesbaden, 107-135, 2018.

Pott, A., Rass, C., und Wolff, F.: Was ist ein Migrationsregime? Eine Einleitung, in: What is a Migration Regime?, Herausgeber: Pott, A., Rass, C., und Wolff, F., Springer, Wiesbaden, 35, 1-16, 2018.

Pütz, R. und Rodatz, M.: Kommunale Integrations- und Vielfaltskonzepte im Neoliberalismus. Zur strategischen Steuerung von Integration in deutschen Großstädten, Geograph. Z., 101, 166183, 2013.

Ratković, V.: Migration und Flucht als postmigrantische Normalität. Fluchtdiskurse abseits des Mainstreams, Migration. Bildung. Frieden, in: Perspektiven für das Zusammenleben in der postmigrantischen Gesellschaft, Herausgeber: Gruber, B. und Ratković, V., Waxmann, Münster, 117-130, 2017.

Robinson, J.: Ordinary Cities. Between Modernity and Development, Routledge, London, 2006.

Rodatz, M.: Migration in diese Stadt ist eine Tatsache. Urban politics of citizenship in der neoliberalen Stadt, Suburban, 2, 35-58, 2014.

Römhild, R.: Jenseits ethnischer Grenzen. Für eine postmigrantische Kultur- und Gesellschaftsforschung, in: Nach der Migration. Postmigrantische Perspektiven jenseits der Parallelgesellschaft, Herausgeber: Yıldı, E. und Hill, M., Transcript, Bielefeld, 37-48, 2015.

Römhild, R.: Beyond the bounds of the ethnic. For postmigrant cultural and social research, J. Aesthet. Cult., 9, 69-57, 2017.

Saunders, D.: Arrival City: How the Largest Migration in History is Reshaping Our World, Random House, Verlag Heinemann, London, 2010 .

Spielhaus, R.: Studien in der postmigrantischen Gesellschaft. Eine kritische Auseinandersetzung, in: Kongressdokumentation. 4 . Bundesfachkongress Interkultur_DIVERCITY_Hamburg_26.-28.10.2012, Hamburg, 96-100, Herausgeber: Kulturbehörde der Freien und Hansestadt Hamburg, online aufrufbar: http: //www.bundesfachkongress-interkultur-2012.de/dokumentation/ (letzter Zugriff: 30 Oktober 2019), 2014.

Spivak, G.: Subaltern Studies: Deconstructing Historiography, in: In Other Worlds - Essays in Cultural Politics, Herausgeber: Guha, R. und Spivak, G., Oxford University Press, Oxford, 197-221, 1988.
Stock, M.: Der Geschmack der Gentrifizierung. Arabische Imbisse in Berlin, transcript, Bielefeld, 2013.

Supik, L.: Dezentrierte Positionierung: Stuart Halls Konzept der Identitätspolitiken, in: Kultur und soziale Praxis, transcript, Bielefeld, online aufrufbar: https://nbn-resolving.org/urn:nbn: de:0168-ssoar-393591 (letzter Zugriff: 30 Oktober 2019), 2005.

Tsianos, V. S.: Homonationalismus und new metropolitan mainstream Gentrifizierungsdynamiken zwischen sexuellen und postsäkularen Politiken der Zugehörigkeit, Suburban, 2, 59-80, 2014.

Tsianos, V. S. und Ronneberger, K.: Panische Räume, Stadtbauwelt, 193, 41-49, 2012.

Vertovec, S.: Super-diversity and its implications, Ethnic Racial Stud., 30, 1024-1054, https://doi.org/10.1080/01419870701599465, 2007.

Vertovec, S.: Talking around super-diversity, Ethnic Racial Stud., 42, 125-139, https://doi.org/10.1080/01419870.2017.1406128,2017.

Wessendorf, S.: Commonplace diversity: social relations in a superdiverse context, Palgrave Verlag, Basingstoke, New York, https://doi.org/10.1057/9781137033314, 2014.

Wiest, K. und Kirndörfer, E.: Paradoxe Aushandlungen von Migration im Diskurs um die Leipziger Einbahnstraße, Raumforschung Raumordnung, 77, 5, https://doi.org/10.2478/rara-20190030, 2019.

Wise, A. und Noble, G.: Convivialities: An Orientation, J. Intercult. Stud., 37, 423-431, 2016.

Yıldız, E.: Stadt ist Migration, in: Eigensinnige Geographien, Herausgeber: Bergmann, M. und Lange, B., VS Verlag für Sozialwissenschaften, Wiesbaden, 2011.

Yıldız, E.: Postmigrantische Perspektiven. Aufbruch in eine neue Geschichtlichkeit, in: Nach der Migration, Postmigrantische Perspektiven jenseits der Parallelgesellschaft, Herausgeber: Yıldız, E. und Hill, M., Transcript, Bielefeld, 19-48, 2015.

Yıldız, E.: Das strategische Geflecht von Migration, Ethnizität und Geschlecht, in: Österreichische Zeitschrift für Soziologie, 41, 29-45, 2016. 